\title{
Correction to "What is a fractional derivative?" by Ortigueira and Machado [Journal of Computational Physics, Volume 293, 15 July 2015, Pages 4-13. Special issue on Fractional PDEs]
}

Udita N. Katugampola*

Department of Mathematical Sciences, University of Delaware, Newark, DE 19716, U.S.A.

\begin{abstract}
There is a debate among contemporary mathematicians about what it really means by a fractional derivative. The question arose as a consequence of introducing a 'new' definition of a fractional derivative in [2]. In a reply, Ortigueira and Machado [3] came up with several very important criteria to determine whether a given derivative is a fractional derivative. According to their criterion, the new fractional derivative, called conformable fractional derivative, introduced by Khalil et al. 2 turns out not to be a fractional derivative, but rather a controlled or conformable derivative. In proving the claim the authors in 3] use an example [3, page 6]. It turns out that the explanation given there needs some corrections and it is the sole purpose of this note.
\end{abstract}

Keywords: Fractional derivative, Fractional calculus, Conformable fractional derivative

The authors in [3], consider the following derivative [1, 2] as an example .

$$
T_{\alpha} f(t)=\lim _{\epsilon \rightarrow 0} \frac{f\left(t+\epsilon t^{1-\alpha}\right)-f(t)}{\epsilon}
$$

for $t>0$.

1. The following reason was given to show that the derivative given in $(1)$ does not satisfy the zero order derivative property:

$$
T_{0} f(t)=\lim _{\epsilon \rightarrow 0} \frac{f(t+\epsilon t)-f(t)}{\epsilon}=\lim _{\epsilon \rightarrow 0} \frac{f[(t(1+\epsilon)]-f(t)}{1+\epsilon} \cdot \lim _{\epsilon \rightarrow 0} \frac{1+\epsilon}{\epsilon} .
$$

It is noted in the same reference that the first factor in $\mathrm{Eq}(2)$ is the order 1 quantum derivative [4, p. 123] given by

$$
D_{q} f(t)=\lim _{q \rightarrow 1^{-}} \frac{f(t)-f(q t)}{(1-q) t}
$$

that coincides with the familiar derivative $\mathcal{D} f(t)$. It turns out that this claim needs to be corrected. In fact, the first factor in question is not a quantum derivative and does not coincide with $\mathcal{D} f(t)$, but rather equal to 0 if the function $f$ is continuous.

${ }^{*}$ Corresponding author. Tel.: +13028312694.

E-mail address: uditanalin@yahoo.com (U. N. Katugampola)

Preprint submitted for publication 
This should be corrected as follows.

$$
T_{0} f(t)=\lim _{\epsilon \rightarrow 0} \frac{f(t+\epsilon t)-f(t)}{\epsilon}=\lim _{\epsilon \rightarrow 0} \frac{f[(t+t \epsilon)]-f(t)}{t \epsilon} \cdot \lim _{\epsilon \rightarrow 0} \frac{t \epsilon}{\epsilon}=\mathcal{D} f(t) \cdot t .
$$

Thus, we see that the first factor in Eq (3) is not a quantum derivative and it does coincide with the derivative $\mathcal{D} f(t)$ taking $t \epsilon=\epsilon^{\prime}$. So $T_{0} f(t)=\mathcal{D} f(t) \cdot t$, which violates the zero order derivative property, that is, we should instead have $T_{0} f(t)=f(t)$.

2. The authors in [3], also show that the derivative (1) does not satisfy the index law, that is, $T_{\alpha} T_{\beta} f(t) \neq$ $T_{\alpha+\beta} f(t)$ for any $\alpha$ and $\beta$ showing that

$$
T_{\alpha} T_{\beta} f(t)=\lim _{\epsilon \rightarrow 0} \frac{f\left[t+\epsilon t^{1-\alpha}+\left(\epsilon t^{1-\beta}\right)^{1-\beta}\right]-f\left(t+\epsilon t^{1-\beta}\right)-f\left(t+\epsilon t^{1-\alpha}\right)+f(t)}{\epsilon^{2}} \neq T_{\alpha+\beta}(t) .
$$

This statement has some errors and should be corrected as follows:

$$
T_{\alpha} T_{\beta} f(t)=\lim _{\epsilon \rightarrow 0} \frac{f\left[t+\epsilon t^{1-\beta}+\epsilon t^{1-\alpha}+O\left(\epsilon^{2}\right)\right]-f\left(t+\epsilon t^{1-\beta}\right)-f\left(t+\epsilon t^{1-\alpha}\right)+f(t)}{\epsilon^{2}} \neq T_{\alpha+\beta} f(t) .
$$

The justification of Equation (5) can easily be seen as follows. Using the definition of the conformable derivative 1 and assuming that all the limits exist, we have

$$
\begin{aligned}
T_{\alpha} T_{\beta} f(t) & =\lim _{\epsilon_{1} \rightarrow 0} \frac{T_{\beta}\left(t+\epsilon_{1} t^{1-\alpha}\right)-T_{\beta}(t)}{\epsilon_{1}} \\
& =\lim _{\epsilon_{1} \rightarrow 0} \frac{1}{\epsilon_{1}}\left[\lim _{\epsilon_{2} \rightarrow 0} \frac{1}{\epsilon_{2}}\left[f\left(t+\epsilon_{1} t^{1-\alpha}+\epsilon_{2}\left(t+\epsilon_{1} t^{1-\alpha}\right)^{1-\beta}\right)-f\left(t+\epsilon_{1} t^{1-\alpha}\right)\right]-\lim _{\epsilon_{3} \rightarrow 0} \frac{1}{\epsilon_{3}}\left[f\left(t+\epsilon_{3} t^{1-\beta}\right)-f(t)\right]\right] \\
& =\lim _{\epsilon_{1} \rightarrow 0} \lim _{\epsilon \rightarrow 0} \frac{1}{\epsilon \epsilon_{1}}\left[f\left(t+\epsilon_{1} t^{1-\alpha}+\epsilon\left(t+\epsilon_{1} t^{1-\alpha}\right)^{1-\beta}\right)-f\left(t+\epsilon_{1} t^{1-\alpha}\right)-f\left(t+\epsilon t^{1-\beta}\right)+f(t)\right],
\end{aligned}
$$

letting $\epsilon=\epsilon_{2}=\epsilon_{3}$ in the last step. This is permitted since "the sum of limits equals the limit of sums".

Now note that

$$
\begin{aligned}
\epsilon\left(t+\epsilon_{1} t^{1-\alpha}\right)^{1-\beta} & =\epsilon t^{1-\beta}\left(1+\epsilon_{1} t^{-\alpha}\right)^{1-\beta} \\
& =\epsilon t^{1-\beta}\left(1+\epsilon_{1}(1-\beta) t^{-\alpha}+\cdots\right) \\
& =\epsilon t^{1-\beta}+\epsilon \epsilon_{1}(1-\beta) t^{1-\alpha-\beta}+\cdots \\
& =\epsilon t^{1-\beta}+O\left(\epsilon \epsilon_{1}\right)
\end{aligned}
$$

Thus we have,

$$
T_{\alpha} T_{\beta} f(t)=\lim _{\epsilon_{1} \rightarrow 0} \lim _{\epsilon \rightarrow 0} \frac{1}{\epsilon \epsilon_{1}}\left[f\left(t+\epsilon_{1} t^{1-\alpha}+\epsilon t^{1-\beta}+O\left(\epsilon \epsilon_{1}\right)\right)-f\left(t+\epsilon_{1} t^{1-\alpha}\right)-f\left(t+\epsilon t^{1-\beta}\right)+f(t)\right] .
$$

Now, to complete the proof, we only need to show that the last statement is independent of the choice of the increment and may consider the same increment for both limits when the second order derivative exists. This is a standard result in advanced calculus and the interested reader may find a similar result, for example, in Mathematical Analysis, 2nd ed by T.M. Apostol in Exercise 5.24. The proof is done by the L'Hospital rule and Theorem $2.2(6)$ of [2], that is, $T_{\alpha} f(t)=f^{\prime}(t) t^{1-\alpha}$. To this end, we consider the following omitting the higher order terms of $\epsilon$.

$$
\begin{aligned}
\lim _{\epsilon \rightarrow 0} \frac{1}{\epsilon^{2}}[f(t & \left.\left.+\epsilon t^{1-\alpha}+\epsilon t^{1-\beta}\right)-f\left(t+\epsilon t^{1-\alpha}\right)-f\left(t+\epsilon t^{1-\beta}\right)+f(t)\right] \\
& =\lim _{\epsilon \rightarrow 0} \frac{f^{\prime}\left(t+\epsilon t^{1-\alpha}+\epsilon t^{1-\beta}\right)\left(t^{1-\alpha}+t^{1-\beta}\right)-f^{\prime}\left(t+\epsilon t^{1-\alpha}\right) t^{1-\alpha}-f^{\prime}\left(t+\epsilon t^{1-\beta}\right) t^{1-\beta}}{2 \epsilon} \\
& =\frac{1}{2} \lim _{\epsilon \rightarrow 0}\left[\frac{T_{\alpha} f\left(t+\epsilon t^{1-\alpha}+\epsilon t^{1-\beta}\right)-T_{\alpha} f\left(t+\epsilon t^{1-\alpha}\right)}{\epsilon}+\frac{T_{\beta} f\left(t+\epsilon t^{1-\alpha}+\epsilon t^{1-\beta}\right)-T_{\beta} f\left(t+\epsilon t^{1-\beta}\right)}{\epsilon}\right] \\
& =\frac{1}{2}\left[T_{\alpha} T_{\beta} f(t)+T_{\beta} T_{\alpha} f(t)\right] \\
& =T_{\alpha} T_{\beta} f(t) .
\end{aligned}
$$


Now, comparing Equation (6) and Equation (7), we can conclude that the expression for $T_{\alpha} T_{\beta} f(t)$ is independent of the choice of the increments $\epsilon$ and $\epsilon_{1}$. Thus, we have

$$
T_{\alpha} T_{\beta} f(t)=\lim _{\epsilon \rightarrow 0} \frac{1}{\epsilon^{2}}\left[f\left(t+\epsilon t^{1-\alpha}+\epsilon t^{1-\beta}+O\left(\epsilon^{2}\right)\right)-f\left(t+\epsilon t^{1-\alpha}\right)-f\left(t+\epsilon t^{1-\beta}\right)+f(t)\right]
$$

as desired.

\section{Acknowledgement}

The research was partially supported by the Department of Defense (USA) research grant 67459MA-15139-MJ.

\section{References}

[1] D.R. Anderson, D.J. Ulness, Properties of the Katugampola fractional derivative with potential application in quantum mechanics, Journal of Mathematical Physics 56, 063502 (2015).

[2] R. Khalil, M. Al Horani, A. Yousef, M. Sababheh, A new definition of fractional derivative, Journal of Computational and Applied Mathematics, 264(2014) 65-70.

3] M.D. Ortigueira, J.A.T. Machado, What is a fractional derivative?, Journal of Computational Physics, 293(15)(2015) 4-13.

[4] M.D. Ortigueira, Fractional Calculus for Scientists and Engineers, Lecture Notes in Electrical Engineering, Springer, Berlin, Heidelberg (2011). 